\title{
Enhancement of persistent currents due to confinement in metallic samples
}

\author{
V. M. Apel, G. Chiappe and M. J. Sánchez \\ Departamento de Física J. J. Giambiagi, Facultad de Ciencias Exactas y Naturales, Universidad de Buenos Aires. Ciudad \\ Universitaria, 1428 Buenos Aires, Argentina.
}

\begin{abstract}
Confinement and surface roughness (SR) effects on the magnitude of the persistent current are analized for ballistic bidimensional metallic samples. Depending on the particular geometry, localized border states can show up at half filling. These border states contribute coherently to the persistent current and its magnitude is enhanced with respect to their value in the absent of confinement. A linear scaling of the typical current $I_{t y p}$ with the number of conduction channels $M$ is obtained. This result is robust with respect to changes in the relevant lengths of the samples and to the SR. Possible links of our results to experiments are also discussed.
\end{abstract}

PACS 72.23.+R

In recent years, advances in nanotechnology made possible to design mesoscopic samples in which the carriers are confined and mainly scattered by the boundaries of the system [1]. In this situation, where the elastic mean free path is much larger than the system size, the sample can be considered in the ballistic regime in order to compute the relevant transport quantities. In addition, the potential that confines the carriers into the mesoscopic device is not perfectly controlled in experiments. Therefore, even when the bulk disorder is absent, surface roughness (SR) is present and as a consequence the carriers are irregularly scattered at the borders of the sample.

When the sample is threaded by a magnetic flux persistent currents originate [2,3]. Persistent currents (PC) have been studied [4] in confined bidimensional cylindrical systems without SR employing discrete models with periodic boundary conditions in one of the directions.

Without reference to $\mathrm{PC}$, a discrete lattice has been considered recently in order to take into account SR in metallic systems [7]. In the latter model, a kind of superficial disorder was introduced through the absence of hopping to and from $L$ sites chosen at random among the $L^{2}$ sites defining the lattice.

In this letter we study the PC for a model in which the confinement and the SR effects can be simultaneously analized. We consider circular (centred and non-centred) and square bidimensional loops on a discrete square lattice. Therefore the confinement breaks completely the translational symmetry of the underlying lattice. This introduces a qualitative difference with respect to cylindrically shaped systems, in which the translational symmetry is preserved in the azimuthal direction and the two degrees of freedom are decoupled. In addition, the samples studied by us have the geometry of those considered in the experiment of Ref. [8].

Although much work has been done concerning the influence of bulk disorder on persistent currents [9], less is known about the ballistic regime. Our goal is to characterize the effect of the confinement and the SR on the magnitude of the persistent current in clean metallic samples. Confinement effects in the context of orbital magnetism were previously considered for semiconductor devices 10$]$.

The total current can be calculated at zero temperature as $I \sim \partial E / \partial \phi$, with $E$ the total energy of the system and $\phi$ the magnetic flux. The value of $I_{t y p} \equiv \sqrt{\int I^{2} d \phi}$ depends on the number of open channels present in the system, $M$. For free electron models with unbounded energy spectrum it is known that $I_{t y p} \sim \sqrt{M}$, 4,10 . This behavior can be understood taking into account the properties of the crossings (or quasicrossings) that appear in the spectrum when the flux is varied and frustrate the coherent increase of the current [11]. As expected, the same scaling with $M$ holds for tight binding models away from half filling [5]. At half filling and for cylindrically shaped systems, it was found that very particular geometrical configurations of the sample could give rise to coherent contributions of all the channels, but those sharp configurations would be very difficult to obtain in an experimental set up 畞.

We consider a $N \times N$ cluster of the square lattice. Taking a single atomic level per lattice site the Hamiltonian is, $H=\sum_{\mathbf{m}} \epsilon_{\mathbf{m}}|\mathbf{m}><\mathbf{m}|+\sum_{\mathbf{m} ; \mathbf{k}} t_{\mathbf{m}, \mathbf{k}}|\mathbf{m}><\mathbf{k}|$, where $(m, n) \equiv \mathbf{m}$ labels the coordinates of the sites in the lattice. All the lengths are given in units of the lattice constant $a$. The hopping integrals $t_{\mathbf{m} ; \mathbf{m}^{\prime}}$ are restricted to nearest neighbours. Assuming that the vector potential has only azimuthal component we take $t_{\mathbf{m} ; \mathbf{m}+\mathbf{l}}=t \exp \left(i \int_{\mathbf{m}}^{\mathbf{m}+\mathbf{l}} \mathbf{A} \cdot \mathbf{d l}\right)$, where the phase is measured in units of the quantum flux $\phi_{o}$ and $\mathbf{l}$ is a vector that points from the site $\mathbf{m}$ to any of its four nearest neighbours. With the on-site energies, $\epsilon_{\mathbf{m}}$, we simulate the confining potential in order to get the required profile of the sample. We will first focus on the circular centred geometry. As the confining potential and the underlying lattice have different symmetry, SR is present in these samples but the rotational symmetry in $\pi / 2$ is preserved. The lattice parameter $a$ represents a cut off for the allowed wavelengths and therefore the SR will be relevant 

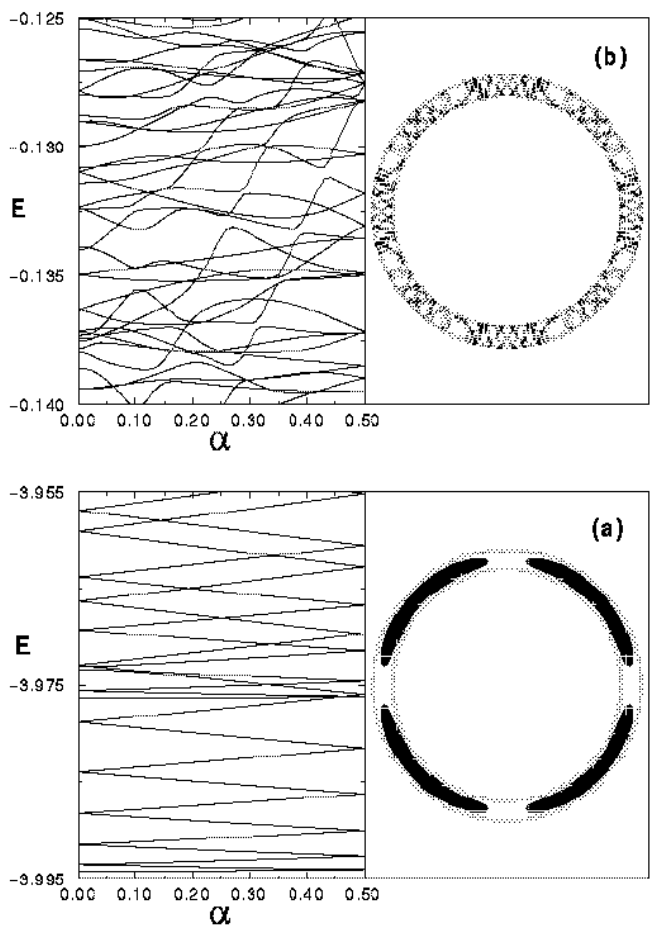

FIG. 1. (a) Energy levels at the bottom of the spectrum as a function of the rescaled magnetic flux $\alpha$ for sample S1 together with a typical charge distribution. (b) Idem as (a) but for the quarter filling region of the spectrum.

near half filling, that is in the metallic regime. The two length scales are chosen as the internal radius $r$ and the width of the sample $W$. While the second determines $M$, the ratio $s \equiv W / r$ controls the strength of SR. For $s>>1$ and for carriers with a wavelength of the order of $a$, two succesive scattering events by the inner and outer boundaries of the sample are almost uncorrelated. On the other hand, for $s<<1$ those events are strongly correlated. Therefore the eigenstates corresponding to these particular wavelengths will evolve from generic extended ones to strongly localized as the value of $s$ is reduced.

In Fig. 11 we show for sample $S 1$ (with $W=40$ and $r=20$ ) the bottom (Fig. 1 (a)) and the quarter filling (Fig. 1 1 (b)) regions of the energy spectrum as a function of the normalized flux $\alpha=\phi / \phi_{0}$ together with a generic charge distribution for each region. Figure 11(a) corresponds to the largest wavelengths, that are the less sensitive to the effect of the SR. This region of the spectrum looks qualitatively similar to the spectrum of an integrable Aharonov-Bohm annular billiard [11]. Moreover, the generic charge distribution corresponds to eigenstates with well defined value of the angular quantum number.

In the quarter filling region the typical wavelengths are larger than $a$ but small enough to make the states sensitive to an effective polygonal boundary. This implies the lost of the continuous symmetry characteristic of the very large wavelengths. Therefore, as the energy rises towards

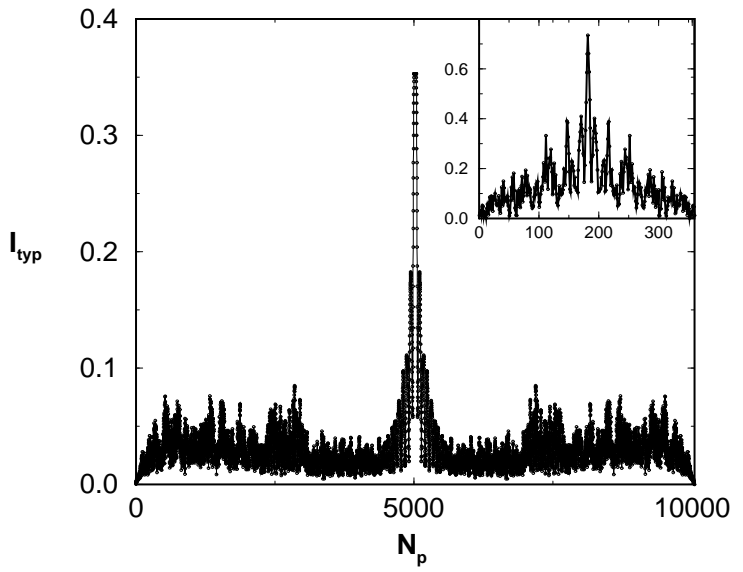

FIG. 2. $I_{t y p}$ as a function of $N_{p}$ for sample $S 1$. Inset: Idem as (a) for a bidimensional square loop with outer square boundary of $20 X 20$ and inner one of $6 \times 6$. Since the mean perimeter of the square loop doubles the mean perimeter of $S 1$ the maximum value of $I_{t y p}$ in the inset is almost twice the value for $S 1$.

half filling, avoided crossings show up. The corresponding charge distributions are quite similar to those for a polygonal billiard and are extended states in general (see Fig. 1(b)).

In any case, when an occuppied level crosses (or quasicrosses) an empty one, the total current $I$ exhibits a discontinuity (or abrupt oscillation) [5]. As it is shown in Fig. 2, below half filling, $I_{t y p}$ is a highly fluctuating function of the number of particles, $N_{p}$. This is a consequence of the random distribution of crossings (or quasicrossings) that appears in these regions of the spectrum [11]. The upper region of the spectrum, close to half filling, is shown in Fig. 3(a) for $S 1$. In this region of energies the wavelengths are of the order of $a$. A bunch of quasi-degenerate states appears at zero flux at half filling which manifestes as a peak in the density of states (DOS) at zero energy. For other circular centred samples a qualitatively similar behavior was found. In a bidimensional sample, a peak in the DOS at zero energy could appear for a very specific relation between the two relevant lengths of the sample. For example, for a cylinder of circumference $L$ (in units of $a$ ) and with $M$ transverse channels the condition is $L=2(p / j)(M+1)$ [ [ 4 , where $p$ and $j$ are coprime integers. For a rectangular sample, i.e with Dirichlet boundary conditions in both directions, with $L \times K$ sites the corresponding formula is $L+1=2(p / j)(K+1)$. As a consequence, for a square shaped sample the above relation is always satisfied and a peak in the DOS manifestes. We expect this result to remain valid in general for the samples considered in this work, in which Dirichlet boundary conditions are imposed on the inner and outer borders, providing the confinement preserves the rotational symmetry in $\pi / 2$. This is confirmed by the numerical results presented in Figs. 1 for the square loops. Therefore, independently of 


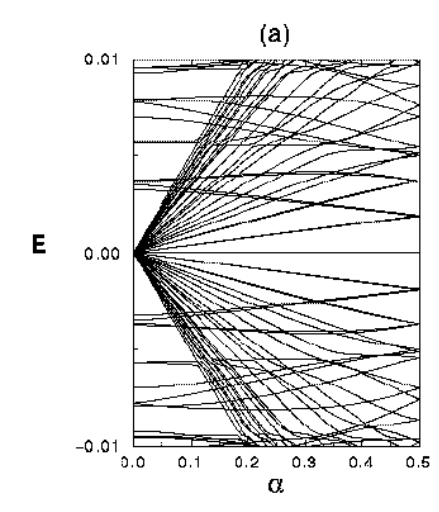

(b)
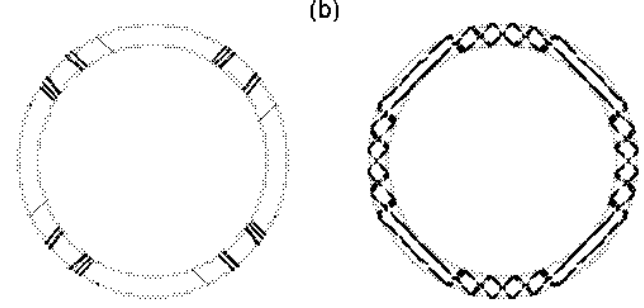

FIG. 3. (a) Energy levels close to half filling as a function of the rescaled magnetic flux $\alpha$ for $S 1$.(b) Typical charge distributions for angularly localized states (left side) and radially localized states (right side).

the number of channels in the loop, the square and centred circular geometries have a peak in the DOS at zero energy. For the circular loops, as a consequence of the $\mathrm{SR}$, the states that conform the peak in the DOS have different characteristics. Some of them are angularly localized states (see the left side of Fig. 3(b)). They are not sensitive to the magnetic flux and correspond to the flat lines in the spectrum. The other type of states are mostly radially localized and look like whispering gallery modes. These states are originated by the correlated scattering with the outer and inner boundary of the sample that allows that the charge distribution does not fill completely the loop and the state remains localized. Althoug localized in one direction, these states go around the sample being sensitive to the magnetic flux and carrying a finite current. Moreover, they have a quasi well defined value of the angular quantum number that characterizes the slope of the eigenenergies as a function of the flux. The right side of fig. 3(b) displays the characteristic charge distributions for these states.

Opposite to what happens for systems with diagonal disorder, in the present model the localization is obtained without introducing an additional scale of energy. Only remains the scale defined by the kinetic energy. At zero flux, the energy of the localized states will be very close to zero as a consequence of the symmetry and the finite character of the spectrum. Therefore, the existence of lo-

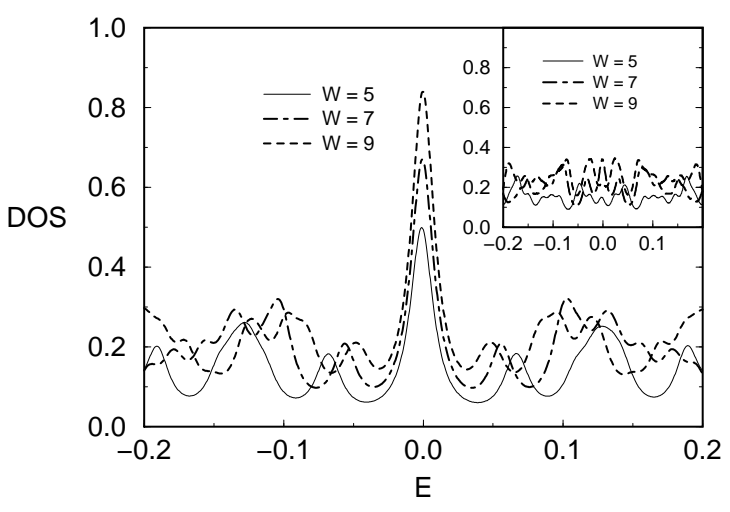

FIG. 4. DOS for square loops with different widths $W$. Notice the peak presents in the DOS at half filling. Inset: DOS for the rectangular samples. There is no bunching of states at zero energy in general.

calized states in the system is manifested as a peak in the DOS that is equivalent to a finite gap in the spectrum of eigenergies. Before estimating the magnitude of the gap we remark that since the eigenenergies are degenerate at $\alpha=0$ but with quite different values of their slopes, each of the radially localized states must belong to a different conduction channel. Being radially localized, these states are quasi one dimensional and the energy gap is estimated as $\Delta_{g}=(\partial E / \partial k) \Delta k \gtrsim(\gamma A)^{-1}$, where $\Delta k$ is the inverse of the mean radius of the ring and $\gamma$ is a finite fraction of $W$ which measures the degree of spatial localization of the state. In order to check the above result we have done numerical calculations for samples with same area $A$, obtaining the same value of $\Delta_{g}$. The gap has an important consequence on the values of $I_{t y p}$ close to half filling. As it is shown in Fig. 2, $I_{t y p}$ increases monotonically with the number of occupied levels (number of particles) $N_{p}$.

Some comments must be made in connection to the non-centred circular loops. Although in this case the SR does not preserve the symmetry in $\pi / 2$, it affects the inner and outer boundary of the samples in the same way. This allows the correlated scattering between the inner and outer borders and therefore angularly and radially localized states also raise up at half filling, as it is illustrated in the spectrum of Fig. 5. In the case of the square loops, although the results are qualitatively similar to those obtained for the circular samples, some important differences must be noticed: $i$ ) The square loops do not have SR and therefore there are not avoided crossings in the spectrum as a function of the flux. ii) Border states carrying a finite current were also found (analogous to the radially localized states of the circular samples). Therefore $I_{t y p}$ increases coherently near half filling as it is shown in the iinset of Fig. 2. iii) As a consequence of 


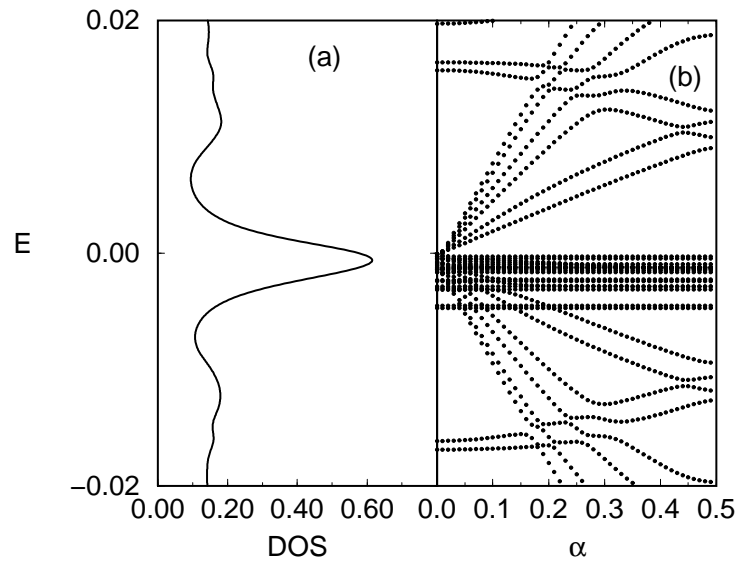

FIG. 5. (a) DOS for a non-centred circular loop of $W=9$. (b) Energy espectrum as function of the flux for the same sample. Flat lines at zero energy are associated with angulary localized states. Flux depending eigenvalues begining at zero energy are associated with radially localized states. The number of the latter (former) increases (decreases) with $W$.

the absence of SR, angularly localized states do not exist at half filling (notice the absence of the plateau in $I_{t y p}$ at half filling).

For rectangular samples the localized border states could occur for very specific relations between it lengths, and in general the spectrum has not bunching of states at zero energy. In other words, a peak in the DOS is not expected at half filling for the rectangular geometry,

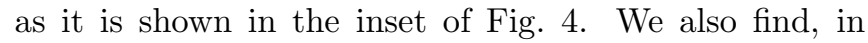
general, that when the localized border states that carry a finite current become dominant over the angularly localized states the scaling of $I_{t y p}$ with $M$ at half filling is substantially enhanced with respect to the known scaling law out of half filling $\left(I_{t y p} \sim \sqrt{M}\right)$. Fig. 6 shows the actual scaling law obtained for the square loops. Notice that the linear increase of $I_{t y p}$ with $M$ is consistent with the fact that each of the localized states bunched at zero energy belongs to a different conduction channel.

The effect described in the present work can help to understand some puzzles about PC in metallic rings. This is the case of the experiment of Ref. [8] in which the $\mathrm{PC}$ of isolated $A u$ rings was measured to be a factor 30 to 100 times larger than the theoretical estimates. In addition, in that experiment the reported value of the signal for the circular loop was 5 times larger than for the rectangular loop (although the predicted theoretical values were roughly the same). Our results, even without providing a definitive explanation of the discrepancies between theory and experiment, are in line with the strong enhancement of the current reported in it and provides the way to explain the observed differences between the circular and the rectangular samples. In fact, as we previously described, for the circular loop we predict a

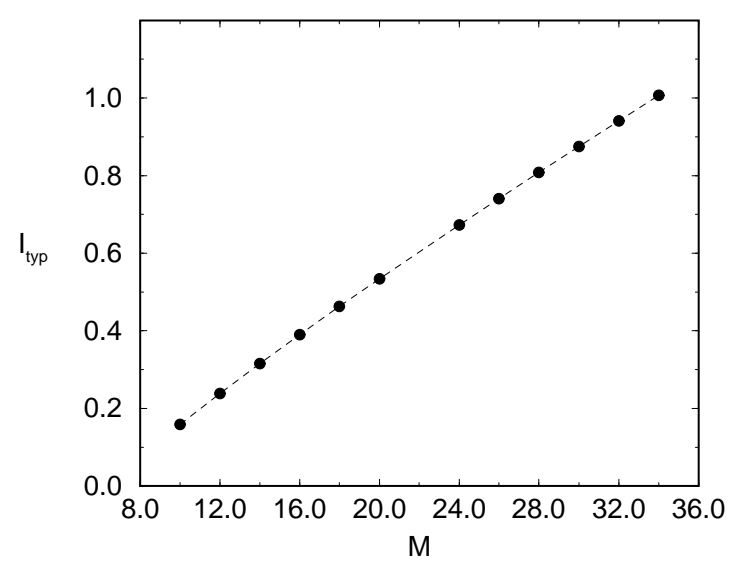

FIG. 6. Linear scaling of $I_{t y p}$ with the number of channels $M$, at half filling, for square loops with the same mean perimeter.

peak in the DOS and therefore the enhancement of the PC. On the other hand, as we already discussed, for the rectangular sample the enhancement is not expected in general. As a last point, we would like to remark that the enhancement of the current predicted in this work does not have a critical dependence on the dimensions and on the SR of the samples, making their experimental realization feasible. Unfortunately we are not aware of new measurements with other sample sizes in order to confirm or not our predictions.

This work was partially supported by UBACYT (TW35-TX61), PICT97 03-00050-01015, CONICET and Fundación Antorchas.

[1] L.P. Kouwenhoven, C. M. Marcus et.al., Proceedings of the NATO Advanced Study Institute on Mesoscopic Electron Transport, edited by L.P. Kouwenhoven, L. Sohn and G. Schön, Kluwer Series E, (1997).

[2] M. Büttiker, Y. Imry and R. Landauer, Phys. Lett. 96 A, 365 (1983).

[3] H. F Cheung, Y. Gefen, E. K. Reidel, and Wei-Heng Shih, Phys. Rev. B 37, 6050 (1988).

[4] H. F Cheung, Y. Gefen and E. K. Reidel, IBM J. Res. Develop.32, 359 (1988).

[5] H. Bouchiat and G. Montambaux, J. Phys. France 50, 2695, (1989).

[6] E. Louis, J. A. Vergés and G. Chiappe, Phys. Rev. B 11, 6912, (1998).

[7] J. A. Vergés and E. Louis, Phys. Rev. B R3803 (1999).

[8] V. Chandrasekar et. al, Phys. Rev. Lett. 67, 3578 (1991).

[9] For a review see T. Guhr, A. Müller-Groeling and H. A. Weidenmüller, Physics Reports 299, 189 (1998).

[10] K. Richter, D. Ullmo and R. Jalabert, Phys. Rep. 276, 
(1996).

[11] A.J. Fendrik and M.J.Sánchez, Eur. Phys. J. B, in press.

cond-mat/9811272. 